\title{
Adaptabilidade e estabilidade em genótipos apomíticos do gênero Paspalum
}

\author{
Adaptability and stability in apomictic genotypes of the genus Paspalum

\begin{abstract}
Emerson André Pereira ${ }^{\mathrm{I}^{*}}$ Miguel Dall'Agnol ${ }^{\mathrm{I}}$ Raquel Schneider $^{\mathrm{I}}$ Roberto Luis Weiler $^{\mathrm{I}}$ Nilo João Ignacio Stork Kuhn ${ }^{I}$ Carine Simioni ${ }^{I}$ Gustavo Mazurkievicz ${ }^{I I}$
\end{abstract} \\ José Antonio Gonzalez da Silva ${ }^{I I}$
}

\section{RESUMO}

O objetivo do trabalho foi analisar a adaptabilidade e estabilidade de genótipos apomiticos do gênero Paspalum frente aos principais caracteres de interesse forrageiro avaliados em diferentes anos e locais. O experimento foi conduzido nos anos de 2010 e 2011 nos municipios de Eldorado do Sul e Augusto Pestana, RS, Brasil. em um delineamento experimental de blocos casualizados com três repetições. As análises de adaptabilidade e estabilidade foram calculadas pelo método Tradicional e pelo Eberhart e Russel. Os genótipos de P. guenoarum Baio e Azulão mostram alta produção de biomassa, mas ajustados a ambientes favoráveis e com reduzida estabilidade. $O$ genótipo de $\boldsymbol{P}$. lepton $28 \mathrm{C}$ evidencia, na massa seca, total adaptabilidade especifica a ambiente desfavorável e, na massa seca de folha, adaptabilidade geral com estabilidade.

Palavras-chave: nativa, melhoramento, genótipo $x$ ambiente, performance.

\section{ABSTRACT}

The objective of this study was to analyze the adaptability and the stability in apomictic genotypes of the genus Paspalum across the main characters of forage interest evaluated in different years and locations. The experiment was conducted in the years 2010 and 2011 in the municipalities of Eldorado do Sul and Augusto Pestana, RS, Brazil, in a randomized complete block design with three replications. Analyses of adaptability and stability were calculated by the Traditional and the Eberhart and Russel method. The genotypes of P. guenoarum Baio anda Azulão shows high biomass production but adapted to favorable environments and reduced stability. The genotypes of P. lepton $28 \mathrm{C}$ show the total dry mass specific adaptability to unfavorable environment and in the dry leaf adaptability with stability.

Key words: native, breeding, genotype $x$ environment, performance.

\section{INTRODUÇÃO}

As mudanças climáticas se tornaram um desafio para os programas de melhoramento de plantas na obtenção de constituições genéticas superiores (ARAUS et al., 2008). Além da busca do progresso genético em cereais e em plantas forrageiras, a maior estabilidade na produção pela redução de perdas por estresses ambientais vem sendo fortemente buscada (VALLE et al., 2008). Nesse contexto, a análise da interação genótipo versus ambiente reflete o efeito genotípico na expressão do fenótipo de forma benéfica ou não pelos estímulos ambientais (CRUZ \& CARNEIRO 2006). Na maioria das vezes, afeta o ganho com a seleção, tornando necessária a estimativa da magnitude e natureza dessa interação (ROSADO et al., 2012). Uma alternativa para amenizar este efeito é a seleção de plantas com ampla adaptação e de elevada estabilidade ou que indique a região mais específica para a máxima expressão do potencial genético da futura cultivar. Para isso, as análises de adaptabilidade e estabilidade estimam o comportamento previsível dos genótipos nos vários ambientes de seleção (CRUZ \& CARNEIRO, 2006; MENDES et al., 2012).

Vários estudos destacam as espécies do gênero Paspalum que, por serem nativas, apresentam vantagens em relação às exóticas pela maior adaptação e grande variabilidade intra e interespecífica em caracteres de interesse forrageiro (PEREIRA et

IDepartamento de Plantas Forrageiras e Agrometeorologia, Universidade Federal do Rio Grande do Sul (UFRGS), 90040-060, Porto Alegre, RS, Brasil. E-mail: emersonpijui@yahoo.com.br. *Autor para correspondência.

IDepartamento de Estudos Agrários, Universidade Regional do Noroeste do Estado do Rio Grande do Sul (UNIJUI), Ijuí, RS, Brasil. Recebido 25.01.13 Aprovado 13.11.14 Devolvido pelo autor 13.05.15 CR-2013-0102.R2 
al., 2012). A maioria dessas espécies apresentam modo de reprodução apomítico e isso implica populações geneticamente homogêneas, inexistindo a recombinação alélica a partir da união de gametas, dificultando assim o ganho genético destas espécies e o lançamento de cultivares. No entanto, com a descoberta de plantas sexuais em espécies naturais de Paspalum, dentre elas, as de Paspalum plicatulum, foi possível criar novos genótipos a partir de cruzamentos com espécies compatíveis (AGUILERA et al., 2011), vislumbrando um novo rumo no melhoramento dessas plantas, antes impossibilitado pela apomixia. Portanto, quando um dos genitores apresenta reprodução sexual, há a possibilidade de formação de variabilidade genética, permitindo a recuperação de progênies elites, com a fixação de alelos na primeira geração de autofecundação pela própria apomixia (ACUÑA et al., 2011). A apomixia, além de manter a presença de blocos gênicos e uma alta uniformidade, permite a contínua exploração da heterose em todas as gerações (CARVALHO et al., 2008). Além disso, as progênies apomíticas, por serem altamente heterozigotas, podem resultar em plantas com maior vigor, produção, estabilidade genética e maior adaptação (FEHR, 1987).

O conhecimento da interação genótipo versus ambiente e da maior adaptação e previsibilidade no comportamento forrageiro de acessos apomíticos do gênero Paspalum pode contribuir na identificação de genótipos promissores para lançamento comercial em curto período de tempo ou no direcionamento de cruzamentos potenciais na obtenção de genótipos mais adaptados, estáveis e produtivos. O objetivo deste trabalho foi analisar a adaptabilidade e estabilidade de acessos apomíticos do gênero Paspalum frente aos principais caracteres de interesse forrageiro avaliados em diferentes anos e locais.

\section{MATERIAL E MÉTODOS}

O experimento foi conduzido nos anos agrícolas de 2009/10 e 2010/11, em Eldorado do Sul e Augusto Pestana, RS, Brasil. A área experimental em Eldorado do Sul está localizada na região da Depressão Central, com coordenadas geográficas de $30^{\circ} 06^{\prime} 02^{\prime \prime} S$ e $51^{\circ} 41^{\prime} 27^{\prime \prime} \mathrm{W}$ e 34 metros de altitude. O solo é classificado como Argissolo Vermelho Distrófico típico e o clima é do tipo Cfa, segundo a classificação de Köppen. A precipitação média anual é em torno de $1400 \mathrm{~mm}$ e a temperatura média anual é de $19,3^{\circ} \mathrm{C}$. Em Augusto Pestana, a área experimental está situada na região Noroeste do estado, com coordenadas geográficas de $28^{\circ} 25^{\prime} 58^{\prime \prime} \mathrm{S}$ e 5400'24'"W e com 290 metros de altitude. O solo é classificado como Latossolo Vermelho Distroférico típico e o clima é do tipo Cfa, conforme Köppen. A precipitação média é em torno de $1600 \mathrm{~mm}$ e a temperatura média anual é de $21,8^{\circ} \mathrm{C}$.

Foram realizadas análises do solo no período que antecedeu a implantação do experimento em ambos os locais. As amostras foram retiradas da camada de 0 a $20 \mathrm{~cm}$ de profundidade e as áreas receberam adubação de base (NPK) na quantidade de 20-150-100 (Eldorado do Sul) e 20-120-100 (Augusto Pestana) e de cobertura com $\mathrm{N}$ na forma de ureia de 180 e $130 \mathrm{~kg} \mathrm{ha}^{-1}$ de nitrogênio em Eldorado do Sul e Augusto Pestana, respectivamente. Houve o fracionamento das adubações de cobertura em seis momentos em cada ano e local, após cada corte, conforme as indicações técnicas para gramíneas perenes de estação quente, seguindo as recomendações da Rede Oficial de Laboratórios de Análise de Solos (2004).

Os acessos utilizados foram obtidos de uma coleção, a partir de coletas realizadas em ecossistemas naturais do bioma Pampa e Planalto Médio do Rio Grande do Sul (REIS et al., 2008; PEREIRA et al., 2011). Anteriormente, foram realizados estudos preliminares e aqueles de maior potencial forrageiro foram selecionados para este estudo: P. lepton (acessos 28B, 26A, 28C, 26D e 28E) e P. guenoarum (acessos Azulão e Baio). Ambas são espécies pertencentes ao mesmo grupo taxonômico, denominado Plicatula. Além disso, foi incluída como testemunha a cultivar 'Pensacola' ( $\boldsymbol{P}$. notatum), que é a única variedade comercial deste gênero disponível no mercado, inclusive, proveniente das condições naturais do Cone sul e apresenta alta relação folha/colmo e qualidade bromatológica (PEREIRA et al., 2011).

Em cada local, utilizou-se o delineamento experimental de blocos ao acaso com três repetições com parcelas foram constituídas por seis linhas de $2,2 \mathrm{~m}$ de comprimento e $0,20 \mathrm{~m}$ de espaçamento. Em cada linha, mudas foram transplantadas a cada $20 \mathrm{~cm}$, o que totalizou 60 plantas por parcela. Foram utilizadas mudas para garantir o estabelicemento dos acessos. Os transplantes ocorreram em 1 e 4 de dezembro de 2009, em Eldorado do Sul e Augusto Pestana, respectivamente. Foram realizados cortes utilizando dois quadrados de $0,50 \times 0,50 \mathrm{~m}$, quando a maioria dos acessos apresentavam altura média das folhas de $35 \mathrm{~cm}$, permanecendo um resíduo de $10 \mathrm{~cm}$ do solo, exceto para a cultivar 'Pensacola', que foi cortada ao atingir altura média de $25 \mathrm{~cm}$ e resíduo de $5 \mathrm{~cm}$, o mais indicado para esta espécie (PEREIRA et al., 2012). Após os cortes, as amostras foram 
levadas ao laboratório para a separação morfológica de folhas, colmos, inflorescências e material morto, secas em estufa de ar forçado a $65^{\circ} \mathrm{C}$ até atingir peso constante. Os caracteres obtidos foram: massa seca total (MST, $\left.\mathrm{kg} \mathrm{ha}^{-1}\right)$, massa seca de folhas (MSF, $\mathrm{kg}$ $\left.\mathrm{ha}^{-1}\right)$ e a relação folha/colmo ( $\left.\mathrm{RFC}=\mathrm{MSF} / \mathrm{MSC}\right)$.

Foi realizada a análise de variância utilizando o teste $\mathrm{F}$, a $5 \%$ de probabilidade para os três fatores (genótipos, anos e locais). Foi efetuada a comparação de médias pelo teste Scott \& Knott a $5 \%$ de probabilidade de erro. Após, foi determinada a estabilidade fenotípica pelo método Tradicional, com base na variação ambiental para cada genótipo, preconizando que aqueles que evidenciam os menores valores de quadrado médio são considerados estáveis (CRUZ et al., 2003). Também foi calculada a estimativa desses mesmos parâmetros pelo método de EBERHART \& RUSSEL (1966), baseado na regressão linear, em que tanto os coeficientes de regressão dos valores fenotípicos de cada genótipo em relação ao índice ambiental, quanto os desvios desta regressão proporcionam estimativas de parâmetros de estabilidade e adaptabilidade, de acordo com o seguinte modelo: $\mathrm{Y}_{\mathrm{ij}}=\beta_{\mathrm{oi}}+\beta_{1 \mathrm{i}} \mathrm{I}_{\mathrm{j}}+\delta_{\mathrm{ij}}+\bar{\varepsilon}_{i j}$, em que: $\mathrm{Y}_{\mathrm{ij}}$ é a média do genótipo i no ambiente ${ }_{\mathrm{j}}^{i j}, \beta_{\mathrm{oi}}$, média geral do genótipo i; $\beta_{1 \mathrm{i}}$, coeficiente de regressão linear, que mede a resposta do i-ésimo genótipo à variação do ambiente; $\mathrm{I}_{\mathrm{j}}$, índice ambiental codificado $(\Sigma \mathrm{I}=0) ; \delta_{\mathrm{ij}}$, desvios da regressão; e $\varepsilon_{i j}$, erro experimental médio. Para a previsibilidade dos genótipos, também foi incluído o recurso auxiliar dos valores do coeficiente de determinação $\left(\mathrm{R}^{2}\right)$. Ressalta-se a importância em aliar a análise de adaptabilidade e estabilidade via regressão com outros métodos, como o Tradicional, fortalecendo as inferências a serem formuladas frente à previsibilidade dos genótipos (CRUZ et al., 2003). As análises foram realizadas pelo programa GENES (CRUZ et al., 2007).

\section{RESULTADOS E DISCUSSÃO}

$\mathrm{Na}$ tabela 1, diferenças significativas foram detectadas em todos os caracteres, tanto para os efeitos principais (genótipo, anos e locais) como os de interação genótipo $\mathrm{x}$ ano, genótipo $\mathrm{x}$ local, ano $\mathrm{x}$ local e genótipo $\mathrm{x}$ ano $\mathrm{x}$ local. Pelos valores de quadrado médio (QM), a magnitude do ano de cultivo foi a fonte de variação mais efetiva em alterar a MST e MSF. Por outro lado, a RFC indicou um maior efeito do genótipo na alteração deste caráter do que a influência do ano. No entanto, as interações A x L e G x A x L mostraram valores de QM superiores aos efeitos principais, indicando as fortes interações sobre a RFC. A MST e a MSF são os principais caracteres utilizados na seleção de plantas forrageiras, bem como a RFC, que permite conhecer a proporção da biomassa total direcionada à produção de folhas e colmos (ASSIS et al., 2008; PEREIRA et al., 2012).

Na tabela 2, em Eldorado do Sul, os acessos de $\boldsymbol{P}$. guenoarum (Azulão e Baio) apresentaram as maiores produções na MST do que os acessos das outras duas espécies nos dois anos de cultivo. Deve-se ressaltar que o segundo ano proporcionou

Tabela 1 - Resumo da análise de variância conjunta de caracteres forrageiros de acessos do gênero Paspalum (P lepton: 28B, 26A, 28C, 26D e 28E; P. guenoraum: Azulão e Baio e P. notatum cultivar 'Pensacola') em dois anos de cultivo em Eldorado do Sul e Augusto Pestana, RS.

\begin{tabular}{|c|c|c|c|c|}
\hline \multirow{2}{*}{ Fonte de variação } & \multirow{2}{*}{ GL } & \multicolumn{3}{|c|}{ 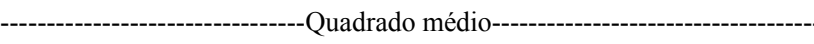 } \\
\hline & & MST $\left(\mathrm{kg} \mathrm{ha}^{-1}\right)$ & $\operatorname{MSF}\left(\mathrm{kg} \mathrm{ha}^{-1}\right)$ & RFC (MSF/MSC) \\
\hline Bloco & 8 & 1032399 & 579238 & 0.59 \\
\hline Genótipo (G) & 7 & $102763861^{*}$ & $58748634^{*}$ & $1181^{*}$ \\
\hline $\operatorname{Anos}(\mathrm{A})$ & 1 & $145679172^{*}$ & $212656579^{*}$ & $114^{*}$ \\
\hline Locais $(\mathrm{L})$ & 1 & $2675086^{*}$ & $761562^{*}$ & $0.05^{*}$ \\
\hline Gx A & 7 & $37182394^{*}$ & $15260521^{*}$ & $6.23^{*}$ \\
\hline Gx L & 7 & $5754648^{*}$ & $3421097^{*}$ & $4.79^{*}$ \\
\hline$A \times L$ & 1 & $19120973^{*}$ & $4630^{*}$ & $1606^{*}$ \\
\hline $\mathrm{G} \times \mathrm{A} \times \mathrm{L}$ & 7 & $3316443^{*}$ & $2159252^{*}$ & $1170^{*}$ \\
\hline Erro & 56 & 1014076 & 446814 & 0.33 \\
\hline Total & 95 & & & \\
\hline Média Geral & & 6401 & 4248 & 2.95 \\
\hline CV (\%) & & 15.73 & 16.20 & 19.42 \\
\hline
\end{tabular}

*Significativo a 5\% de probabilidade do erro; GL, graus de liberdade; MST, Massa Seca Total; MSF, Massa Seca de Folha; RFC, Relação Folha:Colmo; CV, coeficiente de variação. 
maior expressão da variabilidade pela formação de seis classes estatisticamente distintas, mostrando maior performance do genótipo Azulão na espécie P. guenoarum. Além disso, houve superioridade do acesso 28B em relação aos demais em P. lepton. Em Augusto Pestana, a expressão da MST em 2010 mostrou resultado similar a Eldorado do Sul, destacando-se os mesmos acessos de P. guenoarum. Além disso, foi observada a formação de quatro classes fenotipicamente distintas no segundo ano de cultivo, qualificando o genótipo Baio da espécie P. guenoarum e os acessos 26A, 28B, 28C e 28E da espécie P. lepton. Os acessos de $\boldsymbol{P}$. lepton 26A e 28B apresentaram produções de MST similares, independente dos anos e locais de avaliação. A superioridade observada na expressão da MST no segundo ano de cultivo, aliada a melhor detecção da variabilidade no caráter, sugere que seleção de genótipos promissores seja feita a partir do primeiro ano de avaliações. Em espécies perenes de hábito rizomatoso ou estolonífero, o ano de implantação se caracteriza como o período de adaptação das espécies voltado ao estabelecimento inicial. Nos anos seguintes, com a perenização, os fotoassimilados são mais direcionados à expressão dos caracteres forrageiros, facilitando a visualização de diferenças entre os genótipos eficientemente mais produtivos (PEREIRA et al., 2011). Similar a Paspalum, estudo com a espécie perene de verão Brachiaria brizantha indicou que o primeiro ano de cultivo (estabelecimento) foi insuficiente para melhor detecção da variabilidade genética disponível, sugerindo, ao menos, dois anos de avaliação para maior eficiência na seleção de plantas geneticamente superiores (BASSO et al., 2009).

$\mathrm{Na}$ análise da MSF (Tabela 2), resultados similares a MST foram observados, destacando-se os acessos de $\boldsymbol{P}$. guenoarum nos diferentes anos e locais de avaliação, com superioridade do acesso Azulão em relação ao Baio em Eldorado do Sul. Além disso, também foi observada uma maior produção de folha e maior expressão da variabilidade no segundo ano de cultivo, independente das espécies. Destaca-se na espécie P. lepton, em 2011, os acessos 26D e 28B como aqueles que expressaram o melhor desempenho, similar à Pensacola (P. notatum). Em Augusto Pestana, os acessos de $\boldsymbol{P}$. guenoarum foram similares entre si na expressão da MSF nos anos de avaliação e com superioridade aos demais acessos de P. lepton e $\boldsymbol{P}$. notatum. Nessa condição, em 2011, os acessos de $\boldsymbol{P}$. lepton que evidenciaram maior expressão na MSF foram os genótipos 26A e $28 \mathrm{C}$ com produções superiores às apresentadas pela cv. 'Pensacola'. Para a seleção buscando o ganho genético no principal componente ligado à qualidade de forragem (MSF), o segundo ano de avaliação também se mostra mais eficiente, tanto em expressão da produção quanto da variabilidade existente à seleção. No melhoramento de plantas forrageiras, a seleção é feita principalmente sobre o caráter de produção de folhas. Esta estrutura apresenta maior qualidade nutricional e é preferencialmente consumida pelos animais (BRATTI et al., 2009).

No teste de médias para o caráter RFC (Tabela 2), houve variabilidade no primeiro ano de estabelecimento em Eldorado do Sul, destacandose o acesso Azulão de P. guenoarum. Em P. lepton, os genótipos evidenciaram comportamento similar, exceto o acesso 26D, que apresentou desempenho superior na RFC. Em 2011, ano de perenização, comportamento distinto na expressão da RFC foi observado, qualificando a cultivar 'Pensacola' (P. notatum) como a de maior expressão neste caráter. Destaque também é conferido ao acesso 26D, superando, inclusive, os acessos de $\boldsymbol{P}$. guenoarum. Em Augusto Pestana, não foi observada variabilidade no caráter no primeiro ano de avaliação, permitindo a identificação de genótipos superiores apenas no ano de perenização (2011). Em P. guenoarum, destacou-se o genótipo Azulão. Além disso, grande parte dos acessos de P. lepton mostraram comportamento similar para a RFC, semelhante a cultivar 'Pensacola', exceto o genótipo 28B, que obteve um valor médio reduzido. Os dados coletados indicam que a seleção a partir do segundo ano de avaliação (ano de perenização) é o mais indicado. A alta RFC da Pensacola já foi observada em outros estudos, destacando a grande qualidade nutricional desta cultivar (PEREIRA et al., 2011). Ressalta-se que este genótipo é a única cultivar de 'Paspalum' disponível no mercado de sementes no sul do Brasil. Vários autores relatam sobre a importância da relação RFC, considerada um dos mais eficientes caracteres na análise de preferência dos animais em pastejo, inclusive associada à facilidade com que coletam o componente preferido, que é a folha (BRATTI et al., 2009).

Pelo método Tradicional (Tabela 3), os acessos 26A e 28C de P. lepton apresentaram maior estabilidade pelos menores valores de quadrado médio (QM) no caráter MST. Resultado similar também foi obtido pelo método de Eberhart e Russell, que evidenciou previsibilidade no comportamento destes genótipos para este caráter. $\mathrm{O}$ acesso $28 \mathrm{~B}$ apresentou adaptação ampla $\left(b_{1}=0\right)$ e $\quad$ o $26 \mathrm{~A}$ apresentou adaptabilidade específica a ambientes desfavoráveis $\left(b_{1}<1\right)$. Por outro lado, os acessos Azulão e Baio de 
Tabela 2 - Produção de massa seca total (MST), massa seca de folhas (MSF) e relação folha:colmo (RFC) de acessos do gênero Paspalum em dois anos de cultivo e locais.

\begin{tabular}{|c|c|c|c|c|c|c|c|c|c|c|c|c|}
\hline \multirow{3}{*}{$\begin{array}{l}\text { Genótipos } \\
\boldsymbol{P} \text {.guenoarum Azulão }\end{array}$} & \multicolumn{6}{|c|}{------------------Eldorado do Sul------------------ } & \multicolumn{6}{|c|}{ 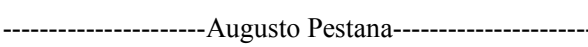 } \\
\hline & \multicolumn{3}{|c|}{----------2010-------- } & \multicolumn{3}{|c|}{----------2011----------- } & \multicolumn{3}{|c|}{----------2010---------- } & \multicolumn{3}{|c|}{--------------2011------------ } \\
\hline & $\mathrm{C}$ & 6519 & $\mathrm{a}$ & $\mathrm{A}$ & 15995 & $\mathrm{a}$ & $\mathrm{C}$ & 8051 & $\mathrm{a}$ & B & 13228 & $\mathrm{~b}$ \\
\hline P. guenoarum Baio & $\mathrm{C}$ & 6267 & $\mathrm{a}$ & $\mathrm{B}$ & 14252 & $\mathrm{~b}$ & $\mathrm{C}$ & 7495 & $\mathrm{a}$ & A & 16837 & $\mathrm{a}$ \\
\hline P. lepton 26A & A & 3894 & $\mathrm{~b}$ & A & 4942 & $\mathrm{e}$ & A & 5526 & $\mathrm{~b}$ & A & 5759 & $\mathrm{c}$ \\
\hline P. lepton 26D & B & 4074 & $\mathrm{~b}$ & A & 6457 & $\mathrm{~d}$ & B & 4866 & $\mathrm{~b}$ & B & 4210 & $\mathrm{~d}$ \\
\hline P. lepton 28B & B & 4529 & $\mathrm{~b}$ & A & 8130 & $\mathrm{c}$ & B & 5384 & $\mathrm{~b}$ & B & 5001 & $\mathrm{c}$ \\
\hline P. lepton $28 \mathrm{C}$ & A & 3660 & $\mathrm{~b}$ & A & 5032 & $\mathrm{e}$ & A & 4594 & $\mathrm{~b}$ & A & 5945 & $\mathrm{c}$ \\
\hline P. lepton $28 \mathrm{E}$ & B & 3554 & $\mathrm{~b}$ & B & 2723 & $\mathrm{f}$ & A & 5904 & $\mathrm{~b}$ & A & 4796 & $\mathrm{c}$ \\
\hline P. notatum $(\mathrm{T})$ & $\mathrm{B}$ & 3953 & $\mathrm{~b}$ & A & 5772 & $\mathrm{~d}$ & $\mathrm{~B}$ & 4444 & $\mathrm{~b}$ & B & 3057 & $\mathrm{~d}$ \\
\hline \multirow[t]{2}{*}{ Genótipos } & \multicolumn{6}{|c|}{---------------------------------------------Massa seca } & de fo & (kg ha & & Pes & ---------' & \\
\hline & \multicolumn{3}{|c|}{---------2010-------- } & \multicolumn{3}{|c|}{----------2011----------- } & \multicolumn{3}{|c|}{ 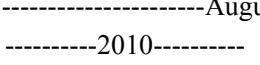 } & \multicolumn{3}{|c|}{-------------2011------------ } \\
\hline P. guenoarum Azulão & $\mathrm{B}$ & 5521 & $\mathrm{a}$ & A & 11219 & $\mathrm{a}$ & $\mathrm{C}$ & 4159 & $\mathrm{a}$ & A & 11171 & a \\
\hline P. guenoarum Baio & $\mathrm{C}$ & 4383 & $\mathrm{~b}$ & $\mathrm{~B}$ & 9850 & $\mathrm{~b}$ & $\mathrm{C}$ & 3973 & $\mathrm{a}$ & A & 12042 & $\mathrm{a}$ \\
\hline P. lepton 26A & $\mathrm{D}$ & 1641 & $\mathrm{c}$ & B & 3443 & $\mathrm{~d}$ & $\mathrm{C}$ & 2827 & $\mathrm{~b}$ & A & 4813 & $\mathrm{~b}$ \\
\hline P. lepton 26D & $\mathrm{C}$ & 2439 & $\mathrm{c}$ & A & 5337 & $\mathrm{c}$ & $\mathrm{C}$ & 2428 & $\mathrm{~b}$ & B & 3521 & $\mathrm{c}$ \\
\hline P. lepton 28B & B & 1877 & $\mathrm{c}$ & A & 4607 & $\mathrm{c}$ & B & 2493 & $\mathrm{~b}$ & A & 3860 & $\mathrm{c}$ \\
\hline P. lepton $28 \mathrm{C}$ & B & 1784 & $\mathrm{c}$ & A & 4075 & $\mathrm{~d}$ & B & 2538 & $\mathrm{~b}$ & A & 4842 & $\mathrm{~b}$ \\
\hline P. lepton $28 \mathrm{E}$ & B & 1490 & $\mathrm{c}$ & B & 1805 & $\mathrm{e}$ & B & 2410 & $\mathrm{~b}$ & A & 3850 & $\mathrm{c}$ \\
\hline P. notatum $(\mathrm{T})$ & B & 2176 & $\mathrm{c}$ & A & 4899 & $\mathrm{c}$ & B & 2018 & $\mathrm{~b}$ & B & 2450 & $\mathrm{~d}$ \\
\hline \multirow[t]{2}{*}{ Genótipos } & \multicolumn{6}{|c|}{ - } & coll & $\mathrm{MSF} / \mathrm{N}$ & Augu & Pes & ------ & $---\cdot$ \\
\hline & \multicolumn{3}{|c|}{---------2010-------- } & \multicolumn{3}{|c|}{-----------2011------------ } & \multicolumn{3}{|c|}{----------2010---------- } & \multicolumn{3}{|c|}{--------------2011------------- } \\
\hline P. guenoarum Azulão & $\mathrm{B}$ & 6.4 & $\mathrm{a}$ & $\mathrm{C}$ & 2.8 & $\mathrm{~d}$ & $\mathrm{D}$ & 1.5 & $\mathrm{a}$ & A & 7.7 & $\mathrm{a}$ \\
\hline P. guenoarum Baio & A & 2.8 & $\mathrm{~b}$ & A & 2.2 & $\mathrm{~d}$ & A & 1.6 & $\mathrm{a}$ & A & 2.8 & $\mathrm{c}$ \\
\hline P. lepton 26A & $\mathrm{B}$ & 1.5 & $\mathrm{c}$ & $\mathrm{B}$ & 2.4 & $\mathrm{~d}$ & $\mathrm{~B}$ & 1.7 & $\mathrm{a}$ & A & 4.9 & $\mathrm{~b}$ \\
\hline P. lepton 26D & $\mathrm{B}$ & 2.2 & $\mathrm{~b}$ & A & 5.1 & $\mathrm{~b}$ & $\mathrm{~B}$ & 1.6 & $\mathrm{a}$ & A & 4.8 & $\mathrm{~b}$ \\
\hline P. lepton 28B & $\mathrm{B}$ & 1.2 & $\mathrm{c}$ & B & 1.5 & $\mathrm{~d}$ & B & 1.2 & $\mathrm{a}$ & A & 3.1 & $\mathrm{c}$ \\
\hline P. lepton $28 \mathrm{C}$ & $\mathrm{B}$ & 1.4 & $\mathrm{c}$ & A & 3.8 & $\mathrm{c}$ & $\mathrm{B}$ & 1.7 & $\mathrm{a}$ & A & 4.2 & $\mathrm{~b}$ \\
\hline P. lepton $28 \mathrm{E}$ & $\mathrm{C}$ & 1.2 & $\mathrm{c}$ & $\mathrm{B}$ & 2.3 & $\mathrm{~d}$ & $\mathrm{C}$ & 1.0 & $\mathrm{a}$ & A & 3.9 & $\mathrm{~b}$ \\
\hline P. notatum $(\mathrm{T})$ & $\mathrm{C}$ & 1.8 & $\mathrm{c}$ & A & 9.2 & $\mathrm{a}$ & $\mathrm{C}$ & 1.3 & $\mathrm{a}$ & $\mathrm{B}$ & 4.1 & $\mathrm{~b}$ \\
\hline
\end{tabular}

Média seguida pela mesma letra maiúscula na linha e minúscula na coluna não diferem entre si pelo Teste de Scott \& Knott, a 5 \% de probabilidade.

P. guenoarum apresentaram adaptação específica a ambientes favoráveis pelos maiores índices de adaptabilidade $\left(b_{1}>1\right)$. Ressalta-se que esses foram os que expressaram maior desempenho forrageiro. No segundo ano, o genótipo Azulão obteve a maior expressão da MST em Eldorado do Sul. Em Augusto Pestana, o acesso Baio se destacou pela maior produção no mesmo período de avaliação. CRUZ et al. (2003) relatam que a adaptabilidade é uma característica herdável e, por isso, sugerem como parâmetro na definição de genitores potenciais. Portanto, os acessos 28B (adaptação ampla), 26A e 28C (estabilidade) de P. lepton e Azulão e Baio (adaptabilidade específica a ambientes favoráveis) de $\boldsymbol{P}$. guenoarum, podem ser indicados às etapas subsequentes para o lançamento como cultivares, além do uso como genitores, demonstrando serem mais ajustados para busca de maior adaptação e estabilidade. CRUZ et al. (2003) comentam que o lançamento de cultivares e a seleção de genitores potenciais para hibridações dirigidas têm por base a boa performance dos genótipos em uma ampla rede de ambientes. No entanto, programas de melhoramento de plantas forrageiras têm encontrado dificuldades na realização de experimentos em vários anos e locais, devido ao baixo retorno econômico, quando comparado aos programas de outras culturas. 
Tabela 3 - Estimativas dos parâmetros de adaptabilidade e de estabilidade fenotípica de caracteres forrageiros de genótipos de Paspalum, segundo o método Tradicional e Eberhart \& Russell.

\begin{tabular}{|c|c|c|c|c|c|}
\hline \multirow{2}{*}{$\begin{array}{l}\left(\text { MST kg ha }{ }^{-1}\right) \\
\text { Genótipos }\end{array}$} & \multirow{2}{*}{$\begin{array}{c}\text { Média } \\
\left(\beta_{0}\right)\end{array}$} & \multirow{2}{*}{$\begin{array}{c}\text { Tradicional } \\
\text { QM }\end{array}$} & \multicolumn{3}{|c|}{ 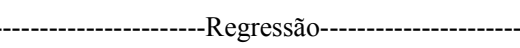 } \\
\hline & & & $\beta_{1 \mathrm{i}}$ & $\mathrm{S}_{\mathrm{di}}^{2}$ & $\mathrm{R}^{2}(\%)$ \\
\hline P. guenoarum Azulão & 10948 & $58684090^{*}$ & $2.83^{*}$ & $1025293^{*}$ & 95.3 \\
\hline P. guenoarum Baio & 11213 & $79147542^{*}$ & $3.06^{*}$ & $6507971^{*}$ & 82.7 \\
\hline P. lepton 26A & 5030 & $2074570^{\text {ns }}$ & $0.34^{*}$ & $312741^{\mathrm{ns}}$ & 78.0 \\
\hline P. lepton 26D & 4902 & $3582964^{*}$ & $0.46^{*}$ & $710400^{*}$ & 41.9 \\
\hline P. lepton 28B & 5761 & $7850410^{*}$ & $0.77^{\mathrm{ns}}$ & $1536253^{*}$ & 52.4 \\
\hline P. lepton $28 \mathrm{C}$ & 4808 & $2706230^{\mathrm{ns}}$ & $0.53^{*}$ & $58209^{\mathrm{ns}}$ & 71.3 \\
\hline P. lepton $28 \mathrm{E}$ & 4244 & $5850616^{*}$ & $-0.22^{*}$ & $2429049^{*}$ & 5.7 \\
\hline P. notatum $(\mathrm{T})$ & 4307 & $3853458^{*}$ & $0.23^{*}$ & $1415938^{*}$ & 9.4 \\
\hline$\left(\mathrm{MSF} \mathrm{kg} \mathrm{ha}^{-1}\right)$ & Média & Tradicional & -------.. & Regressão-- & ------ \\
\hline Genótipos & $\left(\beta_{0}\right)$ & QM & $\beta_{1 \mathrm{i}}$ & $\mathrm{S}_{\mathrm{di}}^{2}$ & $\mathrm{R}^{2}(\%)$ \\
\hline P. guenoarum Azulão & 8017 & $41316772^{*}$ & $2.11^{*}$ & $676639^{*}$ & 96.0 \\
\hline P. guenoarum Baio & 7562 & $48287492^{*}$ & $2.28^{*}$ & $859940^{*}$ & 95.8 \\
\hline P. lepton 26A & 3181 & $5229978^{*}$ & $0.66^{*}$ & $535553^{*}$ & 74.0 \\
\hline P. lepton 26D & 3431 & $5631158^{*}$ & $0.65^{*}$ & $785344^{*}$ & 66.9 \\
\hline P. lepton 28B & 3209 & $4664000^{*}$ & $0.69^{*}$ & $97974^{\mathrm{ns}}$ & 89.6 \\
\hline P. lepton $28 \mathrm{C}$ & 3310 & $5855584^{*}$ & $0.78^{\mathrm{ns}}$ & $48344^{\mathrm{ns}}$ & 93.4 \\
\hline P. lepton $28 \mathrm{E}$ & 2388 & $3284197^{*}$ & $0.32^{*}$ & $1034246^{*}$ & 28.2 \\
\hline P. notatum $(\mathrm{T})$ & 2886 & $5500440^{*}$ & $0.50^{*}$ & $1476622^{*}$ & 41.0 \\
\hline RFC (MSF/MSC) & Média & Tradicional & --------. & egressão--- & --------. \\
\hline Genótipos & $\left(\beta_{0}\right)$ & QM & $\beta_{1 \mathrm{i}}$ & $\mathrm{S}_{\mathrm{di}}^{2}$ & $\mathrm{R}^{2}(\%)$ \\
\hline P. guenoarum Azulão & 4.6 & $25.63^{*}$ & $1.3^{\mathrm{ns}}$ & $8.38^{*}$ & 33.7 \\
\hline P. guenoarum Baio & 2.4 & $0.95^{\mathrm{ns}}$ & $0.3^{*}$ & $0.15^{\mathrm{ns}}$ & 42.5 \\
\hline P. lepton 26A & 2.6 & $7.22^{*}$ & $1.0^{\mathrm{ns}}$ & $0.85^{*}$ & 73.3 \\
\hline P. lepton 26D & 3.4 & $9.81^{*}$ & $1.3^{\mathrm{ns}}$ & $0.38^{*}$ & 89.9 \\
\hline P. lepton 28B & 1.8 & $2.60^{*}$ & $0.6^{*}$ & $0.28^{*}$ & 69.3 \\
\hline P. lepton $28 \mathrm{C}$ & 2.8 & $6.25^{*}$ & $1.0^{\mathrm{ns}}$ & $0.32^{*}$ & 86.1 \\
\hline P. lepton $28 \mathrm{E}$ & 2.1 & $5.06^{*}$ & $0.9^{\mathrm{ns}}$ & $0.18^{\mathrm{ns}}$ & 88.4 \\
\hline P. notatum $(\mathrm{T})$ & 4.1 & $38.91^{*}$ & $1.7^{*}$ & $11.31^{*}$ & 41.2 \\
\hline
\end{tabular}

"significativo a $5 \%$ de probabilidade de erro pelo teste $\mathrm{F}$; ns= não significativo a $5 \%$ de erro pelo teste $\mathrm{F} ; \beta_{0}=\mathrm{Média}$ geral; QM = quadrado médio; $\beta_{1 \mathrm{i}}=$ coeficiente da regressão; $\mathrm{S}_{\mathrm{di}}=$ desvio padrão da regressão; $\mathrm{R}^{2}=$ coeficiente de determinação; $\left(\right.$ Ho: $\left.\beta_{1 \mathrm{i}}=1,0\right)$ e pelo teste $\mathrm{F}$ (Ho: $\left.\mathrm{S}_{\mathrm{di}}^{2}=0\right)$.

Para a MSF, pelo método Tradicional (Tabela 3), os acessos evidenciaram boa performance para ambientes específicos. Destacaram-se pelos menores valores de QM os acessos $28 \mathrm{~B}$ e $28 \mathrm{E}$, porém, este último com menor produção média de MSF. Pelo método Eberhart e Russel, para o mesmo caráter, foram observados que todos os acessos indicaram adaptação a ambientes específicos, exceto o acesso 28C, que apresentou adaptação geral e estabilidade. Ressalta-se que os acessos $28 \mathrm{~B}$ e $28 \mathrm{E}$ evidenciaram estabilidade com adequada produção de folhas. GAMA \& HALLAUER (1980) sugerem que, na seleção de genótipos superiores, deveria ser associada a performance dos caracteres agronômicos com a análise de adaptabilidade e estabilidade, após obtenção de um grupo elite de genótipos.

No modelo proposto por Eberhart e Russel para o caráter RFC (Tabela 3), todos os acessos de $\boldsymbol{P}$. lepton evidenciaram adaptação geral, de maior responsividade às melhorias de ambiente, exceto o acesso 28B, que apresentou adaptabilidade específica a ambientes desfavoráveis. Já o acesso 28E, que apresentou um adaptação geral, também evidenciou estabilidade, porém, com menor expressão da RFC neste estudo. Os acessos de P. guenoarum Azulão e Baio evidenciaram comportamentos distintos, o primeiro por evidenciar adaptação geral sem estabilidade e o segundo com adaptabilidade a ambientes desfavoráveis 
com estabilidade. Resultado similar também obtido pelo método tradicional. Para os demais acessos pelo método Tradicional, indicou instabilidade no caráter. A seleção de genótipos mais ajustados aos ambientes agrícolas com os benefícios da apomixia pode acelerar o lançamento de constituições genéticas superiores, hipótese apoiada pela alta uniformidade, contínua exploração da heterose (CARVALHO et al., 2008) e pela seleção de progênies elite com os caracteres já fixados na primeira geração de autofecundação (ACUÑA et al., 2011).

\section{CONCLUSÃO}

Os genótipos de $\boldsymbol{P}$. guenoarum Baio e Azulão mostram alta produção de biomassa, mas ajustados a ambientes favoráveis e com reduzida estabilidade. O genótipo de $\boldsymbol{P}$. lepton $28 \mathrm{C}$ evidencia na massa seca total adaptabilidade específica a ambiente desfavorável e, na massa seca de folha, adaptabilidade geral com estabilidade.

\section{AGRADECIMENTOS}

Ao Conselho Nacional de Desenvolvimento Científico e Técnológico (CNPq) e ao Associação Sul-Brasileira para o Fomento de Pesquisas em Forrageiras (SulPasto).

\section{REFERÊNCIAS}

ACUÑA, C.A. et al. Tetraploid bahiagrass hybrids: breeding technique, genetic variability and proportion of heterotic hybrids. Euphytica, v. 179, p. 227-235, 2011. Disponível em: <http:// www.researchgate.net/publication/226075553>. Acesso de: 9 mar. 2012. doi: 10.1007/s10681-010-0276-y

AGUILERA, P.M. et al. Interspecific tetraploid hybrids between two forage grass species: sexual Paspalum plicatulum and apomictic P. guenoarum. Crop Science, v.51, n.4, p.1544-1550, 2011. Disponível em: <http:www.crops.org/publications/cs/article $\mathrm{s} / 51 / 4 / 1544$ ?highlight $=\&$ search-result=1>. Acesso em: 8 set. 2013. doi: $10.2135 /$ cropsci2010.10.0610.

ARAUS, J.L et al. Breeding for yield potential and stress adaptation in cereals. Critical Reviews in Plant Science, v.27, p.377-412, 2008. Disponível em: <http://www.tandfonline.com/ doi/pdf/10.1080/07352680802467736>. Acesso de: 3 de abril 2013. doi: $10.1080 / 07352680802467736$.

ASSIS, G.M.L. et al. Seleção de genótipos de amendoim forrageiro para cobertura do solo e produção de biomassa aérea no período de estabelecimento utilizando-se metodologia de modelos mistos. Revista Brasileira de Zootecnia, v.37, n.11, p.1905-1911, 2008.

BASSO, K.C. et al. Avaliação de acessos de Brachiaria brizantha Stapf e estimativas de parâmetros genéticos para caracteres agronômicos. Acta Scientiarum Agronomy, v.31, n.1, p.17-22, 2009. Disponível em: <http://www.scielo.br/scielo. php?pid $=$ S 1807-86212009000100004\&script $=$ sci_arttext $>$. Acesso em: 1 jan. 2013. doi: 10.4025/actasciagron.v31i1.6605.

BRATTI, L.F.S. et al. Comportamento ingestivo de caprinos em pastagem de azevém e aveia-preta em cultivo puro e consorciado. Ciência Animal Brasileira, v.10, p.397-405, 2009.

CARVALHO, F.I.F. et al. Estimativas e implicações da herdabilidade como estratégia de seleção. Pelotas: UFPel, 2001. 99p.

CRUZ, C.D. Programa GENES: aplicativo computacional em genética e estatística. Viçosa: UFV, 2007. 442p.

CRUZ C.D et al. Modelos biométricos aplicados ao melhoramento genético V2, 3 ed., Viçosa. UFV, 2003. 480p.

EBERHART, S.A.; RUSSELL, W.A. Stability parameters for comparing varieties. Crop Science, v.6, 1966. p.36-40.

FEHR, W.R. Principles of cultivar development. New York: Macmillan, 1987. 525p.

GAMA, E.E.F.; HALLAUER, A.R. Stability of hybrids produced from selected lines of Maize. Crop Science, v.20, p.623-626, 1980. Disponível em: $<$ https://dl.sciencesocieties.org/publications/ cs/abstracts/20/5/CS0200050623>. Acesso em: 28 abr. 2012. doi:10.2135/cropsci1980.0011183X002000050019x.

MENDES, F.F. et al. Adaptability and stability of maize varieties using mixed model methodology. Crop Breeding and Applied Biotechnology, v.12, p.111-117, 2012.

PEREIRA, E.A. et al. Produção agronômica de uma coleção de acessos de Paspalum nicorae Parodi. Revista Brasileira de Zootecnia, v.40, p.498-508, 2011. Disponível em: <http://www. scielo.br/scielo.php?pid=S1516-35982011000300006\&script $=$ sci arttext>. Acesso em: 13 mar. 2013. doi:10.1590/S151635982011000300006 .

PEREIRA, E. A. et al. Variabilidade genética de caracteres forrageiros em Paspalum. Pesquisa Agropecuária Brasileira, v. 47, p. 1678-1540, 2012. Disponível em: <http://seer.sct.embrapa.br/ index.php/pab/article/view/12647/8150>. Acesso em:13 dez. 2013.

REIS, C.A.O et al. Chromosome numbers, meiotic behavior and pollen fertility in a collection of Paspalum nicorae Parodi accessions. Crop Breeding and Applied Biotechnology, v.8, p.212-218, 2008. Disponível em: <http://www.sbmp.org.br/cbab/ siscbab/uploads/c8129493-ba4f-9050.pdf >. Acesso em: 1 mar. 2010. doi: 10.12702/1984-7033.v08n03a05.

ROSADO, A.M. et al. Seleção simultânea de clones de eucalipto de acordo com produtividade, estabilidade e adaptabilidade. Pesquisa Agropecuária Brasileira, v.47, n.7, p.964-971, 2012. Disponível em: <http://dx.doi.org/10.1590/ S0100-204X2012000700013>. Acesso em: 11 dez. 2012. doi: 10.1590/S0100204X2012000700013.

COMISSÃO DE QUÍMICA E FERTILIDADE DO SOLO-RS/ SC. Manual de adubação e calagem para os estados do Rio Grande do Sul e Santa Catarina. 10.ed. Porto Alegre: SBCS/CQFS, 2004. 400p.

VALLE, C.B. do et al. Melhoramento genético de Brachiaria. In: RESENDE, R.S. et al. Melhoramento Genético de Forrageiras Tropicais. Campo Grande: Embrapa, 2008. p.13-53. 